\title{
Presentación del Tema Central
}

\author{
Migración Cualificada \\ Presentation of the Central Theme \\ Skilled Migration
}

\begin{abstract}
Cristóbal Mendoza*
Departamento de Sociología

Universidad Autónoma Metropolitana-Iztapalapa

Ciudad de México, México

orCid: http://orcid.org/oooo-0ooI-8694-6830

DoI: http://dx.doi.org/10.28928/revistaiztapalapa/842018/ptc/mendozaperezc
\end{abstract}

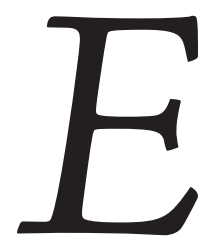

1 tema central del número 84 de Iztapalapa. Revista de Ciencias Sociales y Humanidades recoge y profundiza algunos ejes de discusión del congreso internacional Espacios, Lugares y Territorios: Miradas Latinoamericanas, organizado por la Licenciatura en Geografía Humana de la Universidad Autónoma Metropolitana-Iztapalapa en noviembre de 2016. En dicho contexto, se reunieron equipos de investigación en torno al tema "Más allá del paradigma de las nuevas movilidades: patrones de movilidad y migración en América Latina". El conjunto de investigaciones que se integran aquí tuvo uno de sus puntos de partida en ese debate.

Los trabajos se centran en el estudio del fenómeno de la migración de profesionistas de alta cualificación en América Latina y el sur de Europa. Subyace a los planteamientos la reflexión sobre la idoneidad de los paradigmas de interpretación clási-

*cmp@xanum.uam.mx 
cos (por ejemplo, "la fuga de cerebros") y contemporáneos ("las nuevas movilidades") para interpretar los desplazamientos de los grupos de alta cualificación en el territorio. El paradigma de las nuevas movilidades, acuñado por los sociólogos británicos, presupone que las personas, particularmente aquellas con estudios superiores, se mueven en un mundo sin fronteras, donde los territorios son simplemente continuidades de paisajes homogéneos, ignorando, entre otros aspectos, posibles problemas de integración social o laboral. A diferencia de esa perspectiva, aquí se interpreta el fenómeno desde diferentes enfoques (migración laboral, migración por amor, derechos de ciudadanía, representaciones sociales y espaciales), a partir de experiencias de investigación contrastantes.

La problemática de la migración cualificada ha sido poco estudiada en América Latina. Los análisis desarrollados en esta geografía se han centrado en el "éxodo de cerebros", con énfasis en la vinculación entre los aspectos asociados al desarrollo (o a su falta) y la migración internacional, aunque, bajo el concepto de "intercambio de cerebros", se ha intentado ver algunos aspectos positivos en la creación de capital económico, humano y social en los países de expulsión (véase, por ejemplo, Lozano y Gandini, 20II; Pellegrino, Bengochea y Koolhaas, 20I3). Recientemente, algunos investigadores han explorado temáticas y enfoques alternos a la "fuga de cerebros" y han estudiado la inmigración laboral de alta cualificación en México (Mendoza y Ortiz-Guitart, 2006) o la movilidad académica en América Latina (véase el número monográfico coordinado por Padilla y França, 2015), pero, en todo caso, la migración de alta cualificación constituye todavía una fecunda temática por conocer.

Siguiendo la lógica anterior, la mayoría de pesquisas sobre migración cualificada ha puesto el acento en los desplazamientos hacia el Norte global desde los países considerados menos desarrollados. Es más, cuando la literatura ha abordado el estudio de los flujos hacia los países con menos niveles de bienestar, los desplazamientos se han visto como temporales, dentro de la lógica de los traslados del personal de gestión o técnico de los mercados internos de las empresas transnacionales; un enfoque que, al igual que la "fuga de cerebros", cuenta con una larga tradición en la literatura. En este sentido, nuestro aporte consiste en la revisión de lógicas migratorias diferentes. Se atiende a migraciones permanentes de personas de alta cualificación a México desde el sur de Europa, y en un caso se analizan migraciones Norte-Norte.

Con los artículos aquí reunidos se pretende, además, visibilizar nuevos actores en los flujos migratorios internacionales. En este sentido, Cristóbal Mendoza, del Departamento de Sociología de la Universidad Autónoma Metropolitana-Iztapalapa, en el artículo titulado "Migración y movilidad de los trabajadores cualificados extranjeros de las empresas en México", habla de la incorporación laboral de los 
trabajadores de dirección y gestión españoles e italianos de las empresas en territorio mexicano. Contrasta experiencias laborales de "expatriados" (aquellos que se desplazan dentro de los mercados internos de las transnacionales) con trabajadores "independientes" que optan por otros canales de migración/incorporación laboral. Más que centrarse en las políticas de empresa, como plantea la literatura sobre expatriados, el artículo subraya la atracción de México como destino para estos colectivos de alta cualificación que distan mucho de ser homogéneos e incluyen, en estos momentos de crisis económica del sur de Europa, a personas jóvenes en momentos incipientes de sus carreras ( $y$ no solo a exitosos expatriados que se mueven "libremente" en un mundo globalizado).

El artículo analiza diferentes aspectos de la inserción y la movilidad laborales de estos trabajadores en México y, a partir de estas experiencias, contrapone y, en algunos casos, rebate rasgos dominantes en los paradigmas de interpretación que dominan en la literatura de migración cualificada. Así, se centra en aspectos que la literatura científica ha soslayado a la hora de abordar el análisis de los flujos migratorios de estos inmigrantes. En primer lugar, se discute el papel de México como destino permanente de profesionales, en un contexto de globalización de la economía mundial y de crisis económica. Se apunta que, en México, gran parte de los entrevistados pueden desarrollar sus carreras de tal forma que algunos deciden permanecer en el país. También se aborda el papel del Estado mexicano a la hora de entender los flujos migratorios cualificados. El interés de esta perspectiva radica en que generalmente se obvian las políticas migratorias en el caso de los países con menos niveles de desarrollo, suponiendo que estos no ponen trabas a la libre entrada de profesionales. En el caso de México, el cambio de ley migratoria en 2012 y la llegada de personas jóvenes huidas de la crisis económica han comportado la existencia de inmigrantes de alta cualificación irregulares, sin papeles, trabajando en el país.

El autor también examina las condiciones laborales de aquellos que han llegado bajo el paraguas de los mercados internos de las transnacionales y las contrasta con las de los trabajadores "independientes". Se exponen, de esta manera, una variedad de situaciones y condiciones laborales que apuntan hacia una lectura mucho más compleja que la visión común de la literatura sobre expatriados. Finalmente, se aborda un aspecto poco explorado, relativo a los conflictos en el puesto de trabajo, derivados de culturas empresariales o laborales diferentes. Este asunto, aparentemente menor, es de gran importancia para algunos expatriados que manifiestan su deseo de regresar a su país de origen al no poder adaptarse al medio laboral local.

De esta forma, se arroja luz sobre el fenómeno descrito en un país que no ha sido considerado en la literatura como atractivo para el colectivo de alta cualificación. 
Observando la realidad múltiple de este grupo en México, se intenta complejizar el debate excesivamente enfocado en los países con mayor nivel de desarrollo y desde perspectivas y esquemas de interpretación rígidos. La migración a México parece responder a una doble dinámica de expulsión, motivada por la crisis económica, y atracción, debido a las demandas específicas de algunos sectores de actividad y empresas en el país.

De la misma manera, el texto de Barbara Staniscia, del Departamento de Estudios Europeos, Americanos e Interculturales de la Universidad de Roma La Sapienza, titulado "La movilidad internacional de los jóvenes italianos altamente calificados: motivaciones, experiencias, expectativas y planes de futuro", visualiza un colectivo de jóvenes universitarios que no encuentra empleos ajustados a sus formaciones académicas en Italia y opta por la migración internacional. El caso italiano es sumamente particular no solo por tratarse de un país miembro de la OCDE, sino porque es la cuarta economía más importante en la Unión Europea. Los resultados expuestos en este artículo se basan en entrevistas en profundidad realizadas con quince jóvenes italianos universitarios. En concreto, el artículo aborda las características personales e individuales de los jóvenes migrantes, las principales causas y motores de la movilidad, así como sus experiencias y expectativas de futuro.

A diferencia de lo que señala la literatura que hace énfasis en los motivos económicos para entender la migración internacional de jóvenes, la autora plantea que, en el caso de los italianos, el impulso a salir de su país está determinado por factores psicológicos y culturales: el deseo de vivir experiencias nuevas, de enriquecer el bagaje cultural con conocimiento tanto explícito como tácito, de salir de un país que se considera inadecuado desde el punto de vista de la transparencia y la meritocracia, de ponerse a la prueba y de ver reconocidas las propias capacidades y valorado el talento. Los entrevistados se caracterizan por personalidades no temerosas ante el riesgo, fuertemente curiosas, no ansiosas, abiertas a las novedades y al cambio, y, en algunos casos, de carácter claramente ambicioso.

La crítica a la situación económica y social italiana es inevitable y se hace hincapié en lo que ese país podría ser y no es, en las potencialidades no desplegadas, en los recursos no oportunamente utilizados. Se mira hacia el futuro con optimismo, abiertos a distintas opciones laborales, ya sea en Italia o en otros lugares, aunque los jóvenes confirman una visión pesimista del país. Se trata de una mirada que se ha ido acentuando a partir de la crisis económica de 2008, pero que forma parte, en cierta manera, de la historia del centro y del sur de Italia. Dicha visión se basa en algunos datos objetivos - el alto índice de desempleo juvenil, la ineptitud de la administración pública, la ausencia de meritocracia, la difusión del clientelismo-y 
se fortalece gracias a elementos subjetivos como una tendencia de los italianos de las regiones del centro y del sur a quejarse sobre el sistema, a la indulgencia frente a sí mismos y a una visión positiva sobre los "demás lugares", la xenofilia y una especie de "envidia territorial". Es el contexto sociocultural, pues, el que parece alentar a los jóvenes altamente calificados a encaminarse hacia la movilidad internacional. En este sentido, en las entrevistas, no se señala el "fracaso" de la experiencia migratoria como causa de regreso al lugar de origen. El regreso está motivado básicamente por motivos familiares. La experiencia internacional a menudo se "idealiza", se percibe como una situación sublime, casi un sueño, en la que solo cabe destacar elementos positivos.

El tema de la crisis económica está latente en ambos artículos y, de nuevo, es una materia no analizada en los estudios de migración cualificada, donde este colectivo parece ajeno a los vaivenes económicos que parecen solo afectar a los trabajadores de baja o nula cualificación. Este par de documentos ponen de manifiesto, además, cuestiones relacionadas con el desempleo estructural y la falta de opciones laborales en los países del sur de Europa, que empuja a aquellos jóvenes universitarios con mayor espíritu de aventura a buscar empleo en otros países.

En vez de nuevos colectivos de inmigrantes, el texto de Luciana Gandini, del Instituto de Investigaciones Jurídicas de la UNAM, titulado "Migración de alta educación, desarrollo y... derechos humanos ¿̇la incógnita de la ecuación?”, visualiza nuevas problemáticas poco discutidas en la literatura, como la de los derechos humanos en los migrantes de alta cualificación. El artículo plantea que las reflexiones al respecto se ha centrado mayoritariamente en lo que ocurre en los lugares de destino, pero no se ha revisado exhaustivamente el incumplimiento y violación de derechos - reconocidos en marcos nacionales e internacionales - en los lugares de origen. Dicha situación promueve que la migración se dé no como una elección, un derecho humano de toda persona, sino como una última opción. En el caso mexicano, se muestra que, a pesar del enfoque de migración y desarrollo que ha sido dominante en la literatura, no se evidencian políticas de vinculación con la diáspora cualificada que contribuyan al desarrollo nacional. Si bien se han diseñado programas y acciones con dicho propósito, prevalece una concepción que finalmente cuestiona, incluso, el derecho a migrar.

Con base en estas ideas, se cuestiona si el fenómeno de la migración de personas cualificadas puede explicarse a partir del incumplimiento de un conjunto de derechos en el lugar de origen que estaría propiciando la migración. Adicionalmente, plantea la pregunta de si es posible y útil diseñar un marco conceptual amplio que permita establecer vínculos entre recursos humanos cualificados, migración y desarrollo, y diseñar una política integral articulada. Por último, plantea si realmente tiene sen- 
tido pensar en políticas de vinculación con el país de origen, cuando las personas migraron de manera forzada o involuntaria por el incumplimiento de sus derechos.

De esta manera, la autora se pregunta si es posible concebir la migración cualificada como una consecuencia del incumplimiento de derechos, y en tanto tal, una migración no necesariamente voluntaria. En este contexto, pone en entredicho si el concepto de migración forzada es el más idóneo para la interpretación del fenómeno o si, por el contrario, es más conveniente utilizar el de migración involuntaria. Concluye que, más allá de la adhesión a un concepto u otro, el hecho relevante es la (in)voluntariedad como característica definitoria del movimiento que es expresión del (in)cumplimiento de un catálogo mínimo de derechos, lo cual no había caracterizado a los estudios sobre la migración cualificada. A modo de alegato, la autora expone que se deben incorporar los derechos en la ecuación y relevar, de esta forma, el binomio "migración y desarrollo" por la tríada "migración, desarrollo y derechos".

Los siguientes dos artículos trabajan temáticas que han entrado con fuerza en la literatura de migración cualificada, en línea con el giro cultural de la geografía. Este giro ha servido para poner el foco en el sujeto y visibilizar así temáticas, como son los cambios operados en las relaciones de género, más allá de las parejas que acompañan a los varones expatriados; las dimensiones concernientes al amor y las emociones en los procesos migratorios, o la intersección entre el pasado colonial y las prácticas poscoloniales asociadas con el hecho de ser un expatriado, entre otras (Findlay y Cranston, 20I5). Los siguientes trabajos, basados en un amplio trabajo de campo con inmigrantes españoles e italianos en México, abordan, por un lado, las representaciones sociales y espaciales de estos sujetos, que pueden estar relacionadas, en el caso de los españoles, con un pasado colonial y, por otro, el papel de las emociones y el amor a la hora de entender los procesos migratorios de estos colectivos de alta cualificación en México.

En el primero de estos, "Las distancias sociopsicológicas de la migración española e italiana calificada en México", Xavier Oliveras González, del Departamento de Estudios Urbanos y Medio Ambiente de El Colegio de la Frontera Norte, sede Matamoros, analiza la dimensión sociopsicológica de los procesos migratorios a partir de las representaciones espaciales y sociales (re)producidas de un grupo de inmigrantes españoles e italianos sobre el lugar de destino antes y después de establecerse en México. Interpreta los datos desde el enfoque teórico de las distancias cognitiva, afectiva y mental. De ese modo, estudia y compara las representaciones de esta población migrante antes y después de su establecimiento en México, consideradas, en este sentido, como indicadores de las distancias sociopsicológicas mencionadas. 
La evaluación de los cambios (las diferencias y semejanzas) entre las representaciones anteriores y posteriores al traslado a México permiten al autor evaluar si ha aumentado la aprehensión de la realidad, la conexión afectiva y la comprensión de las convenciones y, por lo tanto, si se reducen, matizan o complejizan los estereotipos, prejuicios o valoraciones. Ello constituye un indicador de la incorporación social de los inmigrantes en el país de residencia y, sobre todo, de la apropiación y adaptación de las representaciones sobre México (re)producidos por las poblaciones locales (es decir, sobre sí mismas y sobre su país). A diferencia de la hipótesis de partida, se encontró que el proceso migratorio supone para algunos informantes una reelaboración de las representaciones según la nueva realidad experimentada (reducción de las distancias), mientras que para otros permanecen las representaciones anteriores e incluso se refuerzan (permanencia de las distancias).

Las representaciones de los dos colectivos europeos de alta cualificación en México muestran que, antes de migrar, los informantes compartían tres representaciones básicas (un espacio en blanco, un México tradicional y feliz, y un México poco desarrollado e inseguro), construidos con referentes adquiridos frecuentemente en un contexto de inmovilidad, a través de los medios de comunicación, el cine o las páginas turísticas de la web. Una vez en el país, aquellas representaciones se tornan más complejas, con la incorporación de referentes locales, la matización o reafirmación de los previos, y la adopción / adaptación de las representaciones locales, construidas en México por la población mexicana. De ello, se desprende que la migración no solo supone un desplazamiento geográfico (en este caso desde España e Italia a México), sino también un “viaje” sociopsicológico (cognitivo, afectivo y mental).

De los resultados, se concluye también que la etapa vital correspondiente a la condición de migrante (entendida como fenómeno sociológico y psicológico) parece estar caracterizada por un estado de transitoriedad, donde se mezclan lo propio y lo ajeno, y las representaciones del lugar de origen con los del sitio de llegada. Ello se refleja, por ejemplo, en las comparaciones (a veces explícitas y conscientes, y otras implícitas e inconscientes) entre el lugar de origen y el de destino, y en la matización y el aumento de la complejidad de las representaciones a partir de la experiencia de la "realidad" y de la integración de referentes de las representaciones locales. De igual manera, la transitoriedad se manifiesta en aquellos que confirmaron y reforzaron las representaciones anteriores, es decir, donde no se produjo una reducción de las distancias. Una muestra de ello es el continuo desplazamiento de las representaciones hacia espacios desconocidos o escasamente experimentados, como es el caso de la búsqueda del "México auténtico" en el centro y sur del país por parte de los 
migrantes residentes en el Norte. Partiendo de una representación previa, aquella se reconstruye de acuerdo con una representación local, según la cual el Norte no es auténtico ni puro. Un segundo mecanismo identificado consiste en la voluntad de mantener, quizá consciente o inconscientemente, las distancias sociopsicológicas, de evitar "concluir" el proceso de migración e integración. La transitoriedad, la inconclusión, pueden entenderse en este sentido como una forma de resistencia ante la pérdida de los referentes propios en detrimento de los ajenos.

El tema central cierra con el artículo de Anna Ortiz Guitart, del Departamento de Geografía de la Universidad Autónoma de Barcelona, titulado "Emociones, amor y experiencias migratorias de españoles e italianos cualificados en México". Este artículo aborda el papel que juegan las emociones en la decisión de migrar, permanecer y regresar entre un grupo de migrantes españoles e italianos radicados en México. A partir del análisis de aspectos emocionales involucrados en sus vínculos de pareja, familiares y de amistad, creados tanto en su lugar de origen como en el de destino, la autora apunta que la decisión de migrar desencadena sentimientos encontrados y diversos por parte de aquellas personas que optan por irse a vivir a otro país: dudas frente al cambio, miedo a equivocarse, tristeza y añoranza, reacomodo emocional, alegría al encontrar trabajo, felicidad por la creación de una nueva familia y satisfacción por unas nuevas amistades.

Desde un enfoque de género, se constata que las parejas toman la decisión de mudarse a México más en función del proyecto laboral de los hombres (tanto si son mexicanos como si son españoles o italianos) que del proyecto de sus parejas. A menudo estas últimas, aun teniendo un buen trabajo remunerado en sus países de origen, abandonan sus empleos para que sus esposos (o novios) tengan mejores oportunidades para encontrar un trabajo equivalente a sus cualificaciones académicas en México. En este sentido, la red familiar (padres y hermanos/as) de las mujeres mexicanas resulta ser un motivo de peso para decidir instalarse en México. No solo el vínculo emocional con la familia sino también el apoyo que esta puede proporcionar en el cuidado de los hijos/as aparece como un aspecto a considerar cuando la pareja valora en qué país asentarse. Cuando se tienen hijos/as, el hecho de estar lejos de los abuelos/as o hermanos/as se ve como una desventaja y, de esta forma, la red familiar se revaloriza, ya que puede ayudar no solo a cuidar a los nietos/as mientras los padres trabajan, sino que también puede proporcionar estabilidad emocional. Cabe destacar, entre los resultados, que los expatriados (todos hombres) comentan que, solos, sin su pareja o sus hijos/as, no se habrían trasladado a México, porque, sin la presencia de su familia, su proyecto profesional carecería de sentido. 
La crianza y la educación de los hijos/as adquieren así una importancia central, como uno de los elementos que más se señalan a la hora de decidir permanecer en México o regresar a Europa. En cuanto al retorno, de hecho, surgen opiniones muy dispares. Así, mientras que unos abogan por regresar y hacen referencia a la calidad de vida en sus lugares de origen o a la calidad de la educación pública en Europa, otros, especialmente los expatriados, señalan que las escuelas (privadas) a las que van sus hijos son de gran calidad y una oportunidad para el ascenso social. Una vez instalados en México, la satisfacción laboral y su inserción social son aspectos relevantes para explicar la permanencia de aquellas personas que migraron a México por amor ( $y$ continúan junto a sus parejas iniciales), pero sobre todo para explicar por qué permanecen aquellas que terminaron su relación con la persona por la que decidieron venir a México. Finalmente, y como resumen de los resultados, la autora asegura que las emociones están constituidas dinámicamente a partir de las experiencias individuales en un lugar determinado y que existen consonancias y disonancias emocionales que afectan las experiencias migratorias, el sentido de pertenencia y el bienestar en el lugar.

De esta manera, el tema central del número 84 de Iztapalapa. Revista de Ciencias Sociales y Humanidades cubre un espacio poco explorado en la literatura científica de migraciones cualificadas y, desde nuestro punto de vista, lo hace de forma innovadora. En primer lugar, el recorte geográfico es transgresor en los estudios de migración cualificada en América Latina, al centrarse en migraciones Norte-Sur en tres de los artículos, y un cuarto en la movilidad Norte-Norte, a diferencia del enfoque dominante en esta literatura que se ha centrado abrumadoramente en los desplazamientos Sur-Norte. En segundo lugar, visualiza tipos de desplazamientos (migración permanente) y colectivos de inmigrantes que han recibido poca atención en la literatura (jóvenes recién titulados, trabajadores no expatriados de las empresas). En tercer lugar, explora temáticas y enfoques innovadores, al menos en la literatura sobre migración cualificada en América Latina (representaciones sociales y espaciales, migración por amor). En cuarto lugar, al poner el foco en el sujeto y no solo en los procesos socioeconómicos, en línea con el giro cultural de la geografía, pone de manifiesto que este colectivo dista, con mucho, de ser un grupo homogéneo y que, en algunos casos, particularmente en el de los jóvenes no expatriados, sufre los vaivenes de la crisis económica internacional, experimenta la irregularidad administrativa en los países de destino, puede sufrir discriminación laboral o social e incluso puede ver mermados sus "derechos". Esta imagen última nos hace replantear los esquemas interpretativos del paradigma de las nuevas movi- 
lidades, que presupone desplazamientos no problemáticos, atemporales, en espacios homogéneos fácilmente reconocibles para los colectivos de alta cualificación.

\section{Bibliografía}

Findlay, Allan M.y Sophie Cranston (2015),"What's in a research agenda?: An evaluation of research developments in the arena of skilled international migration", International Development Planning Review, 37(I), pp. 17-3I. DOI: $10.3828 /$ idpr.2015.3

Lozano, Fernando y Luciana Gandini (20II), "Migración calificada y desarrollo humano en América Latina y el Caribe", Revista Mexicana de Sociología, 73(4), pp. 675-713.

Mendoza, Cristóbal y Anna Ortiz-Guitart (2006),"Hacer las Américas: migrantes españoles de alta calificación en la ciudad de México", Documents d'Anàlisi Geográfica, 47, pp. 93-II4.

Padilla, Beatriz y Thais França (20I5),"Número temático: Mobilidade científica $\&$ imigração qualificada”, Forum Sociológico. Série II, 27.

Pellegrino, Adela, Julia Bengochea y Martín Koolhaas (2013), La migración calificada desde América Latina: tendencias y consecuencias, Montevideo, Universidad de la República-Facultad de Ciencias Sociales. 